\title{
A Novel Fatigue Detection Method of Air Traffic Controller Based on Radiotelephony Communication
}

\author{
Guozhuang Pan, Yonggang Yan and Zhiyuan Shen ${ }^{+}$ \\ College of Civil Aviation, Nanjing University of Aeronautics and Astronautics, Nanjing, China
}

\begin{abstract}
With the rapid development of the civil aviation industry, the total number of civil aviation transportation flights has continued to increase. The requirements for safe operations and transportation efficiency have become higher. High-intensity work requirements make it easier for air traffic controllers to become fatigued during their stay. Based on the analysis of the current situation of air traffic controllers in civil aviation in China, a fatigue detection algorithm for air traffic controllers based on radiotelephony communication is proposed. In the proposed algorithm, an ATC voice recording is extracted as the parameters including short-term energy, short-term average amplitude, instruction time, short-term average power and short-term average amplitude difference. The extracted parameter are used to form fatigue detection model. Then the mapping relationship between the control voice data and the fatigue state is formed based on the quantified fatigue value of the matched controllers. Finally, based on the training and test data from Air traffic management Bureau at Shandong province, the efficiency of the proposed method is verified.
\end{abstract}

Keywords: air traffic controller, fatigue detection, radiotelephony communication, acoustic characteristic.

\section{Introduction}

According to the Statistical Bulletin of Civil Aviation Industry Development 2016, Civil Aviation Administration of China, China's civil aviation industry completed a total transport turnover of 96.251 billion tons of kilometers, passenger turnover of 83.78 billion kilometers, and the national civil aviation airport completed 1.116 billion passenger throughput [1].

The International Civil Aviation Organization (ICAO) defines the fatigue of controllers as a state in which the circadian rhythm is chaotic due to inadequate rest and prolonged wakefulness or the capacity of mental or physical activity is reduced to a certain extent due to excessive workload [2]. This state will impair the ability of civil aviation practitioners to perform their safety duties in terms of their alertness to possible unsafe incidents. The negative effects of fatigue on controllers include narrow attention, distraction, loss of working memory and ambiguous strategies. If the controllers are tired in the process of work, there may be distraction, thus affecting work efficiency, and even leading to unsafe incidents. According to statistics, in 2016, 855,000 irregular flights occurred in the national passenger airlines. Among them, according to Xiamen ATC Safety Reporting System data, $18 \%$ of unsafe incidents are related to the fatigue of controllers [3]. Data from the British Aviation Safety Voluntary Reporting System show that 13\% of operational errors are directly related to controller fatigue, while $21 \%$ are related to fatigue in the US Aviation Safety Reporting System's confidential incident reports [4].

Therefore, it is one of the priority issues to solve the controller's fatigue problem that timely detection. This paper researches the characteristics of controller's work content and analyzes the controller's radiotelephony communications. Based on the physiological changes in the controller's normal and fatigue conditions, we get the controller's quantitative fatigue values and. Finally, we simulate and get beneficial results.

\section{Fatigue Detection Parameters}

Corresponding author. Tel.: +86 13951916587

E-mail address: shenzy@nuaa.edu.cn 
The voice data used in this paper are from a control front-line unit in China. The occurrence time of voice is April 16-20, 2018 and April 27-May 1, 2018. Specifically, it is from 2:00 to 24:00 a day. Each time period has matched the corresponding quantitative fatigue value. Quantitative fatigue value is based on percentile system. The state is judged according to the score. The fatigue is above 60 points, and the awakening is below 60 points. Due to the recording mechanism, the silent band in the channel is automatically eliminated by the system. In the process of speech sampling, the problem of recording murmurs will arise because of environmental factors and acquisition conditions. In order to analyze the controller's land-air calls more conveniently, the speech signal needs to be preprocessed. The pretreatment process is shown in Fig.1.

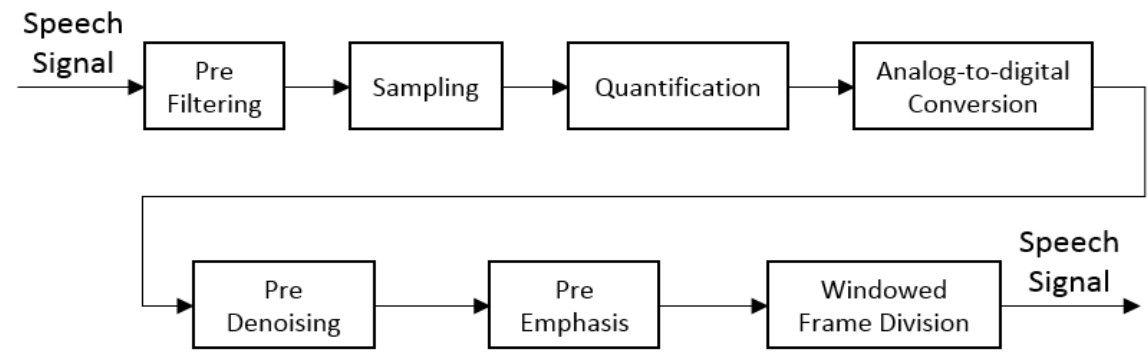

Fig. 1: Speech signal pretreatment process.

Considering the characteristics of the controller's working environment and communication, the unrelated noise mainly concentrates on the environmental noise, so the principle of de-noising is to remove the low-frequency environmental noise in the audio signal. Because of the limited length of the sound signal, FIR high-pass filter is selected for noise reduction [5]. $100 \mathrm{~Hz}$ is chosen for passband interception and $80 \mathrm{~Hz}$ for stopband interception. Frame segmentation is to segment the controller's voice signal [6]. Generally speaking, the frame length of speech signal is 10-30 Ms. In this paper, the data is recorded by CD-ROM with a sampling rate of $44100 \mathrm{~Hz}$. Considering the requirements of fatigue detection, the speech signal frame length in this paper is $30 \mathrm{~ms}$ and the sample speech segment frame number is 1350 frames. In addition, the right object should be selected in the time window of the control call, which is the stage of voice call for the controller. When processing the sample data, the time when the controller starts the voice call is defined as the time when the signal crosses zero and the short-term energy is obviously higher than the environmental noise energy. The time when the signal crosses zero and the short-term energy approaches the environmental noise energy is defined as the time when the controller ends the voice call.

In the process of intercepting voice segments of controlled conversation, there will be leakage of spectrum energy. In order to concentrate the leaked energy on the main lobe and prevent the excessive distortion of the controlled speech signal, the rectangular window can be used to concentrate the main lobe of the rectangular window compared with other window functions. Define the rectangular window as $w(n)$ :

$$
w(n)=S_{N}(n)= \begin{cases}1, & 0 \leq n<N-1 \\ 0, & \text { other }\end{cases}
$$

where $N$ is the length of rectangular window. According to the characteristics of the controller's work, it can be considered that the controller's fatigue is a time-dependent characteristic. When the controller is tired at a certain time, the vocal cord muscle relaxes, and the short-term energy in the corresponding speech signal will also decrease [7]. At the same time, the short-term average amplitude will also appear a certain degree of attenuation. Define short-term energy as $E(n)$.

$$
E_{n}=\sum_{m=n-N+1}^{n}[x(m) w(n-m)]^{2}
$$

Because the short-term energy is the output of the amplitude value of speech signal under certain conditions (the signal passes through a linear filter whose unit function response is $h(n)$ ), Equation 2 can be converted to:

$$
E_{n}=\sum_{m=-\infty}^{\infty} x^{2}(m) h(n-m)=x^{2}(n) * h(n)
$$

where $h(n)=w^{2}(n)$. However, $E(n)$ is calculated by the sum of squares of the amplitude of speech signal, which is sensitive to the sudden change of energy. For this purpose, the short-term average amplitude function $M(n)$ can be used, which is defined as

$$
M_{n}=\sum_{m=-\infty}^{+\infty}|x(m)| w(n-m)=\sum_{m=0}^{N-1}|x(m)|=|x(n)| * w(n)
$$


The sum of absolute values of signals instead of the sum of squares can be used in the average amplitude function to eliminate the effect of sudden changes in sound energy in some controlled calls.

Short-term average power is the average of short-term energy per unit time. The short-term average power is defined as $N(n)$.

$$
N(n)=\frac{E(n)}{\Delta T}
$$

where $E(n)$ is the short-term energy in the $n t h$ time window and $T$ is the length of time window.

Assuming that the period of a periodic signal $x(n)$ is $T, y(n)$ is defined.

$$
y(n)=x(n)-x(n-t)
$$

When $t=a T$, a is an integer, the value of $y(n)$ is 0 . For the whole definition field of $n$, when $n$ takes the integer multiple of the signal period value, $|y(n)|$ should have the minimum value, so the short-term average amplitude difference AMDF is defined as the sum of $|y(n)|$ in a certain interval, that is:

$$
\operatorname{AMDF}_{n}(k)=\sum_{x=n}^{x+N-k-1}|x(x+k)-x(x)|
$$

where $x(n)$ is the signal processed by window function.

\section{Algorithms and Simulation}

There will be individual differences among controllers. It can be judged that different controllers will have different fatigue detection algorithms because of individual differences. The established algorithm needs a lot of statistics and analysis and matches with the controller personally. Therefore, a series of matrix overview algorithms can be established. The calculation fatigue regression model in this paper will refer to ISLSR calculation model. Define controller fatigue regression model as follows:

$$
y=X \beta+\varepsilon
$$

where $y$ is the controller's quantized fatigue value vector, $X$ is the matrix formed by logarithm of each voice parameter detection value (after logarithm, part of the high-order regression can be transformed into a single regression), $\beta$ is the voice parameter vector, and $\varepsilon$ is the random error vector of voice data. The logarithmic value of $X$ can cover part of the higher-order regression function in a certain range, because after logarithmic value, the higher-order independent variable will become a multiplier independent variable.

When judging whether there are multiple collinearity problems, the software can directly read the variance expansion factor and tolerance of each speech parameter, and can also introduce and test the speech parameters one by one and eliminate the undesirable parameters. The least square method is used to find the estimated value of $\beta$, so as to minimize the sum of squares of deviations. Finally, the least square estimation equation of linear regression for speech fatigue is obtained.

$$
\hat{\beta}=\left(X^{\prime} X\right)^{-1} X^{\prime} y
$$

By substituting the results of the above formula into the linear regression model, an individual fatigue algorithm is obtained.

$$
\hat{y}=X \hat{\beta}
$$

The training data is finally intercepted as the voice signal data of the same or similar control instructions issued by the same controller when the control seat is the same. Combined with the field unit investigation and consultation, this paper considers that the controller is awake during the first control period after the change of shift, that is, the voice signal intercepted during the first control period after the change of shift is taken as the index of awake state, and then each of them is taken as the index of awake state. Speech signals in the working period will be used as reference to get the change rate. The change rate is obtained by comparing similar instructions under the same parameters with awake states under different quantified fatigue values. Due to the large number of data results, this paper only shows the processing results during the working period of a controller approaching the seat, and $A 1$ is marked as fatigue during the waking reference working period. The following table shows the change rate of each parameter after taking logarithm from the training data. The change rate of each parameter is the average of the change rate of the same controller under the same quantitative fatigue value, and the number of samples under each quantitative fatigue value is the same. 
Table1: Average variation rate of parameters of the same controller.

\begin{tabular}{cccccccc}
\hline Parameters/Samples & 1 & 2 & 3 & 4 & 5 & 6 & 7 \\
\hline Short time energy & 0 & 39.08 & 45.73 & 36.74 & 50.38 & 50.14 & 50.87 \\
Short time average magni- & 0 & 26.31 & 30.40 & 19.72 & 35.62 & 29.33 & 41.47 \\
tude & & & & & & \\
Instruction duration & 0 & 1.73 & -1.02 & 1.03 & -0.91 & 2.03 & 2.03 \\
Short-term average power & 0 & 10.34 & 39.21 & 21.43 & 26.12 & 32.98 & 31.32 \\
Mean amplitude difference & 0 & 10.34 & 24.23 & 11.33 & 14.12 & 13.35 & 12.62 \\
$\quad$ Fatigue value & - & 77 & 67 & 72 & 92 & 90 & 86 \\
\hline
\end{tabular}

When the value is positive, it means that the short-term energy decreases, the short-term average amplitude decreases, the instruction time increases, the short-term average power decreases, and the short-term average amplitude difference decreases; when the value is negative, it means that the short-term energy increases, the short-term average amplitude increases, the instruction time decreases, the short-term average power increases, and the short-term average amplitude difference increases. In the table, the range of short-term energy change is $36.74 \%$ to $50.87 \%$, with an average decrease of $45.49 \%$; the range of short-term average amplitude change is $19.72 \%$ to $41.47 \%$, with an average decrease of $30.48 \%$; the range of instruction length change is $-1.02 \%$ to $2.03 \%$, with an average increase of $0.82 \%$; the range of short-term average power change is $10.34 \%$ to $39.21 \%$, with an average decrease of $26.90 \%$; the range of short-term average amplitude difference change is $10.34 \%$ to $24.23 \%$, with an average decrease of $14.23 \% .33 \%$. It can be inferred that the controller's five voice characteristics difference decrease from waking to fatigue, and the command length increases or decreases uncertainly.

In regression analysis, it is assumed that the relationship between independent variables and dependent variables is multivariate linear. Independent variables are the rate of change of various parameters of the controller's speech signal, and dependent variables are the controller's fatigue index. In order to construct multiple regression equation, multiple collinearity tests must be carried out. If there is a definite or highly correlated relationship between independent variables, it is said that there are multiple collinearities between independent variables [8]. Multiple collinearity may cause the regression data to deviate from the actual data. The variance expansion factor (VIF) of the five parameters obtained by SPSS analysis is less than 10, and the tolerance is greater than 0.1 . Therefore, it can be determined that there is no multiple collinearity among the five independent variables.

Thus the equation of linear model can be considered:

$$
\mathrm{Y}=21.256+232.2 \ln X_{1}-58.9 \ln X_{2}-145.2 \ln X_{3}-28.1 \ln X_{4}-136.1 \ln X_{5}
$$

where $X 1$ is short-term energy, $X 2$ is short-term average amplitude, $X 3$ is instruction time, $X 4$ is short-term average power, $X 5$ is short-term average amplitude difference.

Then it should turn to replace the data of other controllers and repeat the above steps. The final regression equation is as follows:

$$
\begin{gathered}
Y^{\prime}=53.26+174.8 \ln X_{1}-79.4 \ln X_{2}+15.2 \ln X_{4}-76.5 \ln X_{5} \\
Y^{\prime \prime}=89.24+204.2 \ln X_{1}-80.4 \ln X_{2}-159.2 \ln X_{3}-10.3 \ln X_{4}-96.6 \ln X_{5}
\end{gathered}
$$

The above is the regression equation formed by three controllers approaching the post under the training data. Among them, Y' eliminates the instruction length parameter because of the obvious multi-collinearity.

The following is a comparison between the calculated data of regression equation and the actual data. 


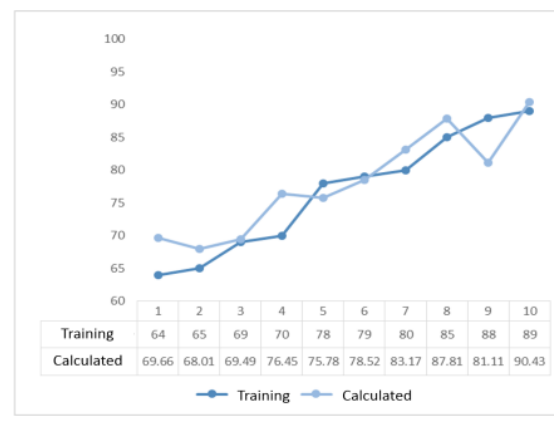

(a)

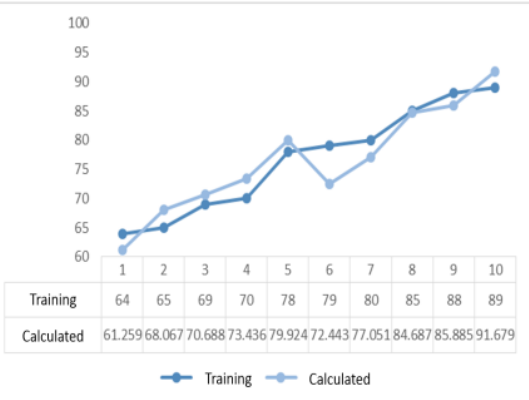

(b)

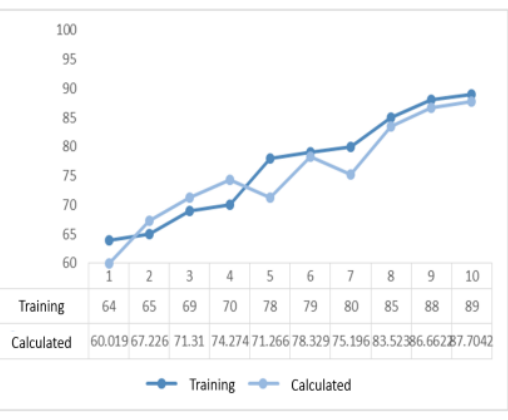

(c)

Fig.2: Y, Y' and Y" regression calculation data and training data. (a) Y; (b) Y'; (c) Y".

\section{Conclusion}

Based on the analysis of the current situation of air traffic controllers in civil aviation in China, a fatigue detection algorithm for air traffic controllers is proposed. In this paper, a 10-day voice recording of a line control unit in China is used as the data source of the detection algorithm. Based on the quantified fatigue value of the matched controllers, the mapping relationship between the control voice data and the fatigue state is formed. A fatigue detection model is proposed. Fatigue detection algorithm is multivariate linear regression algorithm. The parameters are selected. The extraction and processing of each parameter are completed. Multivariate fusion can cover more fatigue data of reaction controllers and avoid over-distortion of test results caused by a single parameter. Combining with the field speech data, the training data is selected to form a personal fatigue detection algorithm, and the test data is selected to verify the fatigue detection algorithm, and the better verification results are obtained.

\section{Acknowledgements}

The authors would like to thank the financial support by the Open Fund of Graduate Innovation Base (Laboratory) of Nanjing University of Aeronautics and Astronautics funded project (kfjj20180724).

\section{Reference}

[1] Civil Aviation Administration of China. Statistical Bulletin of Civil Aviation Industry Development 2016. 2017.5. http://www.caac.gov.cn/XXGK/XXGK/TZTG/201705/P020170508503230814473.pdf.

[2] International Civil Aviation Organization (ICAO). DOC. 9966 Fatigue Risk Management Systems-Manual for Regulation [S]. First edition. ICAO, 2012.

[3] Sun Tao, Chen Yu. Fatigue Management and Prevention in Air Traffic Control [J]. Air Traffic Management, 2005(5): 4-10.

[4] NTSB. Commercial Transportation Operator Fatigue Management Reference [EB/OL]. 2003, 7: 1-1

[5] Wang Peng. Design of High Pass Filter Based on MATLAB [J]. Science and Technology Information in China, 2012(19):85-189.

[6] Liu Qi, Ying Guoxiang. Research on Speech Signal Preprocessing Technology Based on MATLAB [J].Electronic Technology and Software Engineering, 2014(01):62-63.

[7] Feng Kang, Shi Huikun. A Pitch Detection Method with Reference to Short-term Average Energy and Average Amplitude Difference Function [J]. Journal of Huainan Normal University, 2003(05):9-11.

[8] Meng Qinghe. Multiple Collinear Processing in Multivariate Regression Analysis [J].Health Statistics of China, 1997(01):51-52. 